[Chem. Pharm. Bull.

35( 4$) 1619-1623(1987)]$

\title{
Effect of Diethyl Maleate and Sodium Salicylate on Cefmetazole Transport in the in Vitro Rat Everted Intestinal Sac: Influence of $\mathrm{Ca}^{2+}$ and Vanadate in the Media
}

\author{
Takumi SuzuKa, Atsushi Furuya, Akira Kamada, \\ Masaru Yamazaki, and Toshiaki Nishihata* \\ Faculty of Pharmaceutical Sciences, Osaka University, \\ 1-6 Yamadaoka, Suita, Osaka 565, Japan
}

(Received October 1, 1986)

\begin{abstract}
In $\mathrm{Ca}^{2+}$-containing media, $1 \mathrm{~mm}$ diethyl maleate (DEM) increased the in vitro cefmetazole transport in rat everted colonic sac rapidly after a significant nonprotein thiol loss, but the increase in the ileal sac occurred only slowly after a significant nonprotein thiol loss. However, $\mathrm{Ca}^{2+}{ }_{-}$free media promoted the adjuvant effect of DEM rapidly even in the ileal sac. Since vanadate in the $\mathrm{Ca}^{2+}$-containing media also promoted the adjuvant effect of DEM, it is suggested that a perturbation of $\mathrm{Ca}^{2+}$ homeostasis as a secondary effect after nonprotein thiol loss is related to the increase of the intestinal mucosal permeability and that the ileal mucosa has stronger resistance to the secondary effect than the colonic mucosa does. The adjuvant effect of $300 \mathrm{~mm}$ salicylate (SA) was also promoted in $\mathrm{Ca}^{2+}$-free media. Since the effect of SA was stronger than that of DEM even though SA caused less nonprotein thiol loss, it is considered that the effect of SA involves at least two mechanisms.
\end{abstract}

Keywords - cefmetazole; transport; everted intestinal sac; calcium ion; vanadate; diethyl maleate; salicylate; nonprotein thiol

Since it has been reported that salicylate and its derivatives enhance rectal absorption of hydrophilic compounds, ${ }^{1-3)}$ various absorption-promoting adjuvants ${ }^{1,4)}$ have been investigated. ${ }^{5-12)}$ However, salicylate has a greater adjuvant effect at the rectum (including colon) than at small intestine. ${ }^{13)}$ Although it has recently been reported that nonprotein thiol loss caused by diethyl maleate might be related to the increase of intestinal mucosal permeability to cefmetazole, ${ }^{14,15)}$ cefmetazole transport in the presence of diethyl maleate also occurred at the colon more effectively than at small intestine, in spite of no difference of nonprotein thiol loss between the tissues. ${ }^{14)}$ Further, it has been reported that nonprotein thiol loss in hepatocyte induced the liberation of $\mathrm{Ca}^{2+}$ from cells in $\mathrm{Ca}^{2+}$-free medium. ${ }^{16}$ )

In the present report, we examined the effect of $\mathrm{Ca}^{2+}$ in the media on the actions of diethyl maleate and salicylate in increasing the intestinal mucosal permeability to passive transport of cefmetazole, using the in vitro everted intestinal sac method.

\section{Experimental}

Materials-Sodium salicylate (SA) was obtained from Nakarai Chemicals Co., Ltd. (Kyoto, Japan). Diethyl maleate (DEM) and vanadium pentoxide were obtained from Sigma Inc. (St. Louis, U.S.A.) and vanadate was prepared by dissolution of vanadium pentoxide in sodium hydroxide solution. Sodium cefmetazole was supplied by Sankyo Co., Ltd (Tokyo, Japan). Other reagents used were of analytical grade.

Preparation of an Everted Intestinal Sac — Wistar male rats, weighing 200 to $300 \mathrm{~g}$, were fasted for $16 \mathrm{~h}$ prior to experiments. After midline incision of the abdomen of rats anesthetized with sodium pentobarbital ( $30 \mathrm{mg} / \mathrm{kg}$, i.p.). $20 \mathrm{ml}$ of saline was injected from the aorta to rinse blood out of the intestine, and then both the ileum (as a small 
intestinal region) and colon (including the upper rectum) were removed. Each segment was everted. All procedures after excising the intestine were carried out in ice-cold saline saturated with $\mathrm{O}_{2}: \mathrm{CO}_{2}$ gases $(95 \%: 5 \%)$.

Transport Study - The in vitro rat intestinal transport of cefmetazole was studied by the method of Barr and Riegelman. ${ }^{17)}$ Krebs-Ringer III buffer ( $\mathrm{pH} 7.0$ ) (designated as $\mathrm{Ca}^{2+}$-containing medium) was used as the medium, and $\mathrm{Ca}^{2+}$-free buffer $\left(\mathrm{Ca}^{2+}\right.$-free medium) was prepared by addition of equivalent concentrations of sodium chloride instead of calcium chloride in the Krebs-Ringer III buffer. During experiments, the mucosal medium was saturated with $\mathrm{O}_{2}: \mathrm{CO}_{2}$ gases $(95 \%: 5 \%)$. Each everted intestinal sac containing $0.5 \mathrm{ml}$ of medium was immersed in $20 \mathrm{ml}$ of medium containing cefmetazole $(800 \mu \mathrm{g} / \mathrm{ml})$ at $37^{\circ} \mathrm{C}$, and was taken from the medium at designated times. After gentle rinsing of the sac surface 3 times with $50 \mathrm{ml}$ of ice-cold saline, the serosal fluid was collected to measure the amount of cefmetazole in the medium. An intestinal segment was homogenized for assay of nonprotein thiol. Transport of cefmetazole was represented in terms of the clearance rate $\left(C L_{\mathrm{cmz}}\right)$, which was calculated with the following equation.

$$
C L_{\mathrm{cmz}}(\mathrm{ml} / \mathrm{h} / \mathrm{g} \text {-tissue })=(\mathrm{S}) /([\mathrm{M}] \times(\text { incubation period }) \times(\text { wet weight of intestinal sac }))
$$

where $(\mathrm{S})$ represents the cumulative amount of cefmetazole in the serosal medium and $[\mathrm{M}]$ represents the initial concentration of cefmetazole in the mucosal medium.

Assay _ Assay of cefmetazole was carried out by the high-pressure liquid chromatographic method described previously. ${ }^{18}$ Nonprotein thiol in the homogenate was measured by the method of Ellman ${ }^{19)}$ and glutathione was used as a standard compound for nonprotein thiol assay. ${ }^{20)}$

Statistical Analyses_- Statistical analyses were performed by using Student's $t$-test.

\section{Results}

Figures 1 and 2 show the effect of $\mathrm{Ca}^{2+}$ in the media on cefmetazole transport $\left(C L_{\mathrm{cmz}}\right)$
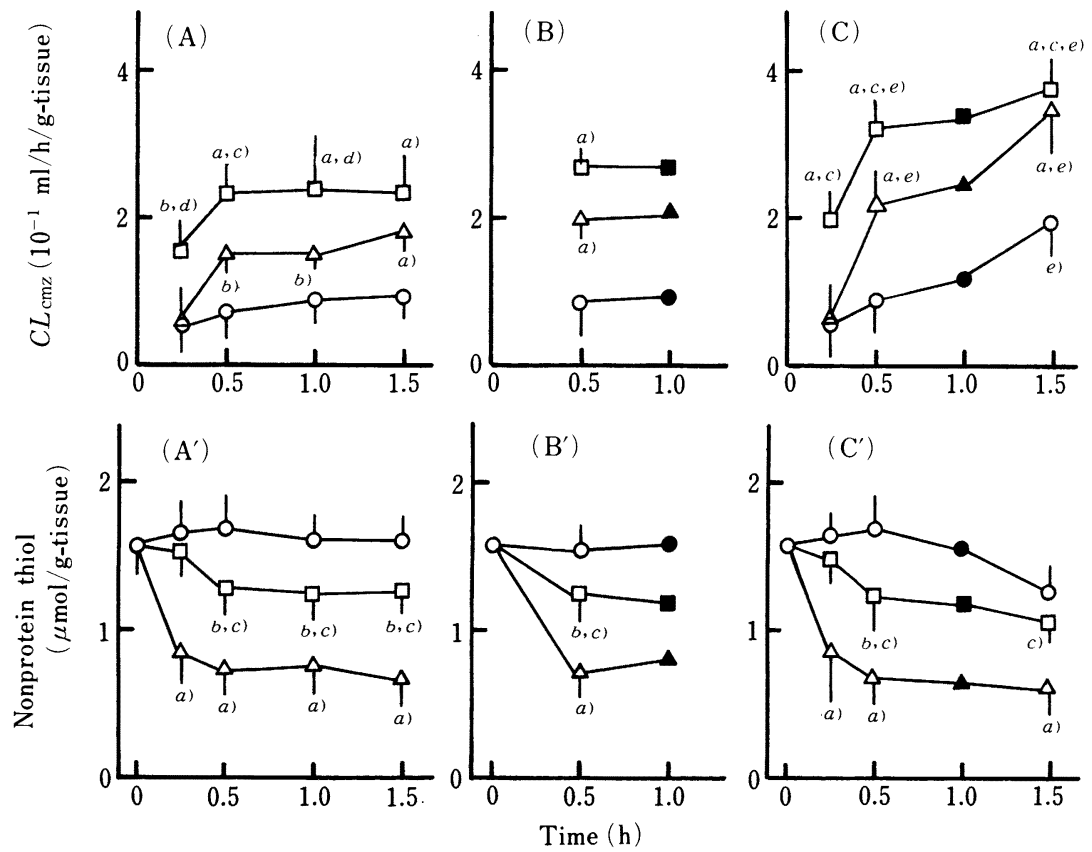

Fig. 1. Effect of DEM (Triangles, $1 \mathrm{~mm}$ ) and SA (Squares, $300 \mathrm{~mm}$ ) on Cefmetazole Transport $\left(C L_{\mathrm{cmz}} ; \mathrm{A}, \mathrm{B}\right.$ and $\left.\mathrm{C}\right)$ as a Function of Incubation Time from the Mucosal Side to the Serosal Side through Rat Everted Colonic Sac, and on Nonprotein Thiol Concentrations in the Tissue $\left(\mathrm{A}^{\prime}, \mathrm{B}^{\prime}\right.$ and $\left.\mathrm{C}^{\prime}\right)$

Circles represent the control (without DEM or SA). $\mathrm{A}$ and $\mathrm{A}^{\prime}, \mathrm{Ca}^{2+}$-containing media; $\mathrm{B}$ and $\mathrm{B}^{\prime}, \mathrm{Ca}^{2+}$-containing media with $2 \mathrm{~mm}$ vanadate; $\mathrm{C}$ and $\mathrm{C}^{\prime}, \mathrm{Ca}^{2+}$-free media. Experimental numbers were more than four for open symbols and one for closed symbols.

Each value represents the mean \pm S.D. a) $p<0.01$ versus control (no additive); $b$ ) $p<0.05$ versus control; $c$ ) $p<0.01$ versus addition of DEM; $d$ ) $p<0.05$ versus addition of DEM; $e$ ) $p<0.01$ versus $\mathrm{Ca}^{2+}$-containing media. 

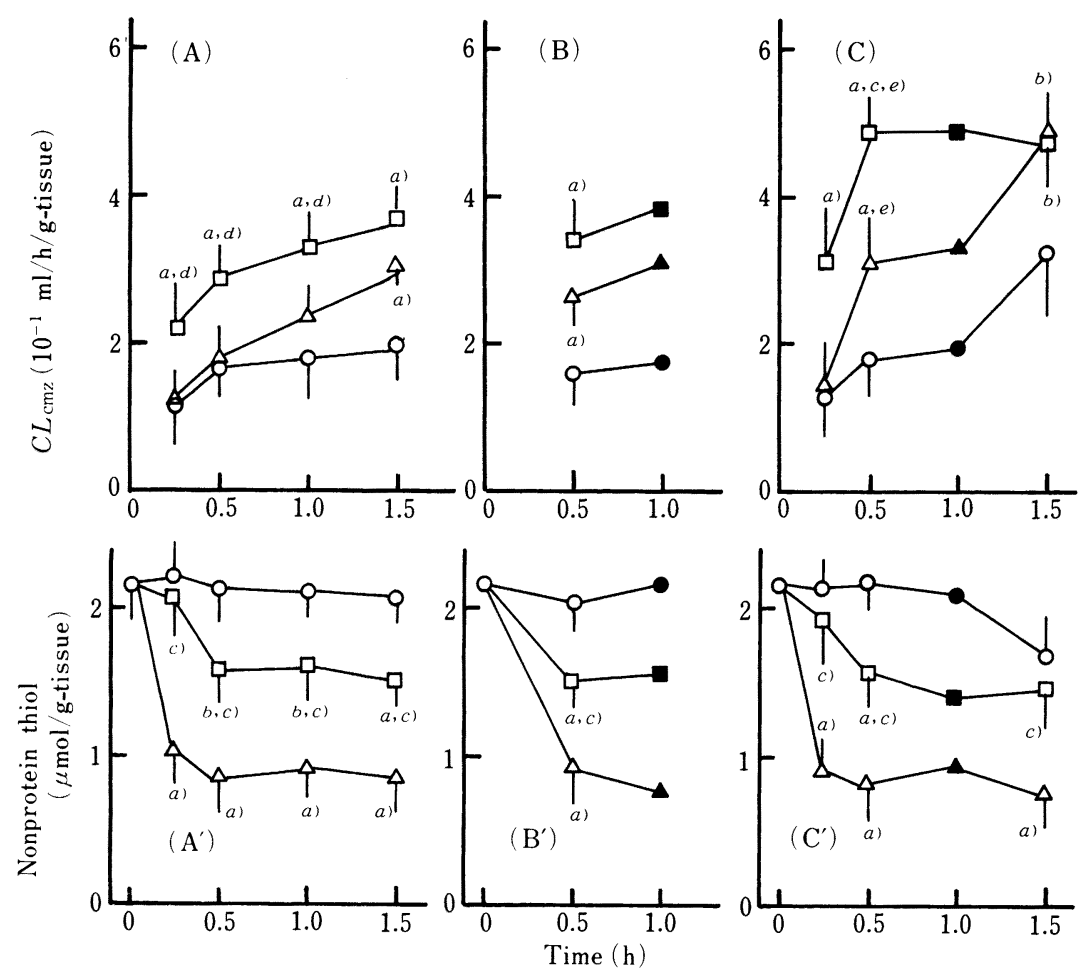

Fig. 2. Effect of DEM (Triangles, $1 \mathrm{~mm}$ ) and SA (Squares, $300 \mathrm{~mm}$ ) on Cefmetazole Transport $\left(C L_{\mathrm{cmz}} ; \mathrm{A}, \mathrm{B}\right.$ and $\left.\mathrm{C}\right)$ as a Function of Incubation Time from the Mucosal Side to the Serosal Side through Rat Everted Ileal Sac, and on Nonprotein Thiol Concentration in the Tissue $\left(\mathrm{A}^{\prime}, \mathrm{B}^{\prime}\right.$ and $\left.\mathrm{C}^{\prime}\right)$

Circles represent the control (without DEM and SA). The media used were as described in Fig. 1. Symbols are the same as in Fig. 1.

Each value represents the mean \pm S.D. a) $p<0.01$ versus control (no additive); $b$ ) $p<0.05$ versus control; $c$ ) $p<0.01$ versus addition of DEM; $d$ ) $p<0.05$ versus addition of DEM; $e$ ) $p<0.01$ versus $\mathrm{Ca}^{2+}$-containing media.

from the mucosal side to the serosal side through the rat everted intestinal sac (Fig. 1 for colonic sac and Fig. 2 for ileal sac). $C L_{\mathrm{cmz}}$ was not influenced by $\mathrm{Ca}^{2+}$ in the media, though a tendency for $C L_{\mathrm{cmz}}$ to increase in the later stage (at $1.5 \mathrm{~h}$ ) was observed in $\mathrm{Ca}^{2+}$-free media.

The addition of DEM (final concentration; $1 \mathrm{mM}$ ) to the mucosal medium caused a significant nonprotein thiol loss, which was not influenced by $\mathrm{Ca}^{2+}$ and vanadate in the media (Fig. $1 \mathrm{~A}^{\prime}$ to $\mathrm{C}^{\prime}$ and $2 \mathrm{~A}^{\prime}$ to $\mathrm{C}^{\prime}$ ). In $\mathrm{Ca}^{2+}$-free media, $C L_{\mathrm{cmz}}$ in the sac of both intestines increased significantly after $0.5 \mathrm{~h}$ of incubation with DEM (Fig. $1 \mathrm{C}$ and $2 \mathrm{C}$ ). The addition of DEM to the $\mathrm{Ca}^{2+}$-containing media resulted in an increase of $C L_{\mathrm{cmz}}$ of the colonic sac after $0.5 \mathrm{~h}$ of incubation (Fig. 1A), but did not increase $C L_{\mathrm{cmz}}$ of the ileal sac until $1 \mathrm{~h}$ after the start of incubation (Fig. 2A). However, the addition of vanadate to the $\mathrm{Ca}^{2+}$-containing media promoted the effect of DEM in increasing $C L_{\mathrm{cmz}}$ of the ileal sac even at $0.5 \mathrm{~h}$ after the start of incubation (Fig. 2B)

The addition of SA (final concentration; $300 \mathrm{~mm}$ ) to the mucosal medium also caused nonprotein thiol loss, which was also not influenced by $\mathrm{Ca}^{2+}$ and vanadate in the media (Fig. $1 \mathrm{~A}^{\prime}$ to $\mathrm{C}^{\prime}$ and $2 \mathrm{~A}^{\prime}$ to $\mathrm{C}^{\prime}$ ). Although the degree of nonprotein thiol loss caused by SA was less than that caused by DEM, the increase of $C L_{\mathrm{cmz}}$ by SA in both $\mathrm{Ca}^{2+}$-containing media and $\mathrm{Ca}^{2+}$-free media was greater than that by DEM. It should be noted here that the increase of $C L_{\mathrm{cmz}}$ by $\mathrm{SA}$ was observed as early as $0.25 \mathrm{~h}$ after the start of incubation, before nonprotein 
thiol loss was observed. The $\mathrm{Ca}^{2+}$-free media also promoted the effect of SA in increasing $C L_{\mathrm{cmz}}$, but vanadate in $\mathrm{Ca}^{2+}$-containing media did not.

\section{Discussion}

Among absorption-promoting adjuvants that enhance intestinal absorption of hydrophilic drugs, ethylenediamine tetraacetic acid (EDTA) increased cefmetazole absorption to similar extents at the colon and small intestine. ${ }^{14)}$ However, it has been reported that the effect of DEM and SA was weak at the small intestine, ${ }^{13,14)}$ as mentioned earlier. EDTA modifies the permeability of the tight junction of intestinal epithelium by removing $\mathrm{Ca}^{2+}$ from the junctional region, ${ }^{21}$ indicating that agents such as EDTA which directly modify the structural components of epithelium may act to similar extents at all intestinal regions. Thus, it may be considered that the adjuvant effect of DEM occurs indirectly.

Although it has been reported that the action of $1 \mathrm{~mm}$ DEM might be related to its effect in causing nonprotein thiol loss, ${ }^{13.15)}$ the effect of DEM in $\mathrm{Ca}^{2+}$-containing media appeared with a long lag time after nonprotein thiol loss, especially in the ileal sac (Fig. 1A, 1A', 2A and $\left.2 \mathrm{~A}^{\prime}\right)$. However, the effect of DEM in increasing $C L_{\mathrm{cmz}}$ in $\mathrm{Ca}^{2+}$-free media appeared rapidly, even in the ileal sac. Thus, the adjuvant effect of DEM seems to be a secondary effect occurring after nonprotein thiol loss, and may be related to $\mathrm{Ca}^{2+}$ homeostasis in the intestinal epithelium. It has been reported that nonprotein thiol loss in hepatocytes caused a significant $\mathrm{Ca}^{2+}$ release from cells along with bleb formation in $\mathrm{Ca}^{2+}$-free medium. ${ }^{16)}$ The results, which show that vanadate (an inhibitor of $\mathrm{Ca}^{2+}$ transport through cells ${ }^{21)}$ ) promoted the effect of DEM, seem to support the idea that a perturbation of $\mathrm{Ca}^{2+}$ homeostasis after nonprotein thiol loss increases the permeability to cefmetazole. The weak effect of DEM on $C L_{\mathrm{cmz}}$ of the ileum in $\mathrm{Ca}^{2+}$-containing media may be due to greater resistance of the ileum to the hypothetical perturbation of $\mathrm{Ca}^{2+}$ homeostasis, in comparison with that of the colon.

The adjuvant effect of $300 \mathrm{~mm}$ SA in increasing $C L_{\mathrm{cmz}}$ was greater in the colonic sac than in the ileal sac. Since the adjuvant effect of $\mathrm{SA}$ in $\mathrm{Ca}^{2+}$-free media was also stronger than that in $\mathrm{Ca}^{2+}$-containing media, the mechanism of SA action in increasing $C L_{\mathrm{cmz}}$ may also involve the perturbation of $\mathrm{Ca}^{2+}$ homeostasis after nonprotein thiol loss. It has been reported that $\mathrm{SA}$ uncouples oxidative phosphorylation to produce adenosine triphosphate (ATP) in mitochondria $^{22)}$ and it is known that synthesis of glutathione requires ATP. Thus, the decrease of nonprotein thiol loss may be due to partial impairment of glutathione synthesis. However, it was observed that the effect of $300 \mathrm{~mm} \mathrm{SA}$ in increasing $C L_{\mathrm{cmz}}$ in both intestinal sacs appeared before nonprotein thiol loss by SA and was greater than that of $1 \mathrm{~mm} \mathrm{DEM}$ in spite of the lesser effect of SA on nonprotein thiol loss. This observation seems to indicate that another mechanism is involved in the effect of SA in increasing $C L_{\mathrm{cmz}}$; it has been suggested that SA increases the membrane permeability by binding to membrane component(s). ${ }^{23,24)}$ Therefore, at least two mechanisms may be involved in the effect of SA in increasing cefmetazole transport.

\section{References}

1) T. Nishihata, J. H. Rytting, and T. Higuchi, J. Pharm. Sci., 69, 744 (1980).

2) T. Nishihata, J. H. Rytting, T. Higuchi, and L. Caldwell, J. Pharm. Pharmacol., 33, 334 (1981).

3) S. Yoshioka, L. Caldwell, and T. Higuchi, J. Pharm. Sci., 71, 593 (1982).

4) L. Caldwell, T. Nishihata, J. Fix, S. Selk, R. Cargil, C. R. Gardner, and T. Higuchi, Meth. and Find. Exptl. Clin. Pharmacol., 6, 503 (1984).

5) A. Kamada, Y. Kohno, H. Yaginuma, M. Saeki, M. Yamazaki, and T. Nishihata, Yakuzaigaku, 45, 188 (1985).

6) A. Kamada, T. Nishihata, S. Kim, M. Yamamoto, and N. Yata, Chem. Pharm. Bull, 29, 2012 (1981).

7) K. Morimoto, T. Takeeda, Y. Nakamoto, and K. Morisaka, Int. J. Pharm., 14, 149 (1982). 
8) K. Morimoto, E. Kajima, T. Takeeda, Y. Nakamoto, and K. Morisaka, Int. J. Pharm., 14, 149 (1983).

9) K. Nishimura, Z. Nozaki, A. Yoshimi, N. Kakeya, and K. Kitao, Jpn. J. Antibiot., 36, 1692 (1983).

10) H. Okada, I. Yamazaki, T. Yashiki, and H. Mima, J. Pharm. Sci., 72, 75 (1983).

11) H. Yaginuma, T. Nakata, H. Toya, T. Murakami, M. Yamazaki, and A. Kamada, Chem. Pharm. Bull., 29, 2974 (1981).

12) T. Nishihata, H. Yoshitomi, and T. Higuchi, J. Pharm. Pharmacol., 38, 69 (1986).

13) T. Suzuka, T. Nishihata, M. Yamazaki, and A. Kamada, Chem. Pharm. Bull., 33, 4600 (1985).

14) T. Nishihata, H. Takahata, and A. Kamada, Pharm. Res., 6, 307 (1985).

15) T. Nishihata, M. Miyake, H. Takahata, and A. Kamada, Int. J. Pharm., 33, 89 (1986).

16) S. A. Jewell, G. Bellomore, H. Thor, S. Orrenius, and M. T. Smith, Science, 210, 1257 (1982).

17) W. Barr and S. Riegelman, J. Pharm. Sci., 59, 154 (1970).

18) T. Nishihata, H. Takahagi, M. Yamamoto, H. Tomida, J. H. Rytting, and T. Higuchi, J. Pharm. Sci., 73, 109 (1984).

19) G. L. Ellman, Arch. Biochem. Biophys., 82, 70 (1959).

20) S. Szabo, J. S. Trier, and P. W. Frankel, Science, 214, 200 (1981).

21) A. L. Palmora, I. Meza, G. Beaty, and M. Cereyide, J. Cell. Biol., 81, 736 (1980).

22) T. M. Brody, J. Pharmacol. Exp. Ther., 117, 39 (1956).

23) T. Nishihata and T. Higuchi, Biochim. Biophys. Acta, 775, 269 (1984).

24) H. Kaji, T. Horie, M. Hayashi, and S. Awazu, Life Sci., 37, 523 (1985). 